\title{
A Study on the Mechanical Properties of the Representative Volume Element in Fractal Porous Media
}

\author{
Jianjun Liu, ${ }^{1,2}$ Mingyang Wu, ${ }^{1}$ Zhengwen Zhu, ${ }^{1}$ and Zuliang Shao ${ }^{1}$ \\ ${ }^{1}$ School of Geoscience and Technology, Southwest Petroleum University, Chengdu, Sichuan 610500, China \\ ${ }^{2}$ State Key Laboratory of Oil and Gas Reservoir Geology and Exploitation, Southwest Petroleum University, Chengdu, \\ Sichuan 610500, China \\ Correspondence should be addressed to Jianjun Liu; liujj0906@163.com
}

Received 3 May 2017; Revised 14 August 2017; Accepted 11 September 2017; Published 18 October 2017

Academic Editor: Micol Todesco

Copyright (C) 2017 Jianjun Liu et al. This is an open access article distributed under the Creative Commons Attribution License, which permits unrestricted use, distribution, and reproduction in any medium, provided the original work is properly cited.

\begin{abstract}
Natural porous structure is extremely complex, and it is of great significance to study the macroscopic mechanical response of the representative volume element (RVE) with the microstructure of porous media. The real porous media RVE is generated by an improved quartet structure generation set (QSGS), and the connectivity of the reconstructed porous media models is analyzed. The fractal dimension of the RVE is calculated by the box-counting method, which considers the different porosity, different fractal dimension, and different mechanical properties of the matrix. Thus, the stress-strain curves of the RVE in the elastoplastic stage under different conditions are obtained. The results show that when the matrix mechanics are consistent, the mechanical properties of the porous media RVE are negatively correlated with the porosity and fractal dimension; when the difference between the porosity and fractal dimension increases, the trend is more obvious. The mechanical properties of the RVE have a positive correlation with the modulus of elasticity of the matrix, though the correlation with Poisson's ratio of the matrix is weak. The fractal dimension of complex porous media can better predict the RVE mechanical characteristics than the porosity.
\end{abstract}

\section{Introduction}

Porous media comprise solid particles bonded to a skeleton, with many tiny, widely distributed voids, such as in a reservoir core $[1,2]$. The porous media structure is complicated, and the mechanical and transmission characteristics of the RVE are greatly influenced by their microstructures. Therefore, the mechanical properties of the RVE are established and revealed the microstructure of the porous media, which is of great theoretical significance and has applicable prospects for studying the multifield coupling problem related to the development of underground fluid. In recent years, with the improvement of scanning technology and computer computing ability, the reconstruction method of the microstructure of porous media has been improved, and the structural characteristics of the porous media RVE and its macroscopic mechanical response have been more intensely studied.

Mesomechanical homogenization methods are used in the study of the mechanical properties of porous media, such as the sparse method, Mori-Tanaka method, self-consistent method, and differential method. Their basic assumption is that the pores are spherical and the pore stiffness is extremely small. However, due to the large difference in pore geometry and the uneven distribution of the spatial location, the prediction accuracy of the RVE is poor when using the mesoscopic mechanical homogenization method.

At present, the study of the RVE characteristics of porous media can be divided into two classes, and one is based on statistical principles. Lu et al. [3] calculated the density, porosity, surface area, and pore size distribution of the porous media RVE, and the macroscopic characteristics of the macroscopic elastic modulus are given. Yakub et al. [4] made porous media with different porosities by using sintered clay and wood chips; the porosity, pore size distribution, and density of the porous media were statistically analyzed by the mercury intrusion method, and the result shows that the compressive strength of the porous media was negatively correlated with porosity. Zhao et al. [5] used the porosity of porous media as the characterization parameter, and the ballast experiment was carried out, the results of which 
show that the compressive strength of the porous medium RVE was negatively correlated with its porosity. Wang et al. [6] considered the statistical characteristics of the pore (the local maximum porosity ratio, average maximum pore ratio increment, and average maximum porosity ratio), and the results of the numerical simulation show that the average pore ratio of the shear zone is linear with the average plastic shear strain in the strain softening stage. Mandelbrot [7] proposed the concept of fractal dimension of a complex structure. Then, Pia et al. [8] combined the fractal theory with statistics to establish a mixed fractal model of the pore size distribution, and the effects of the pore size distribution on the elastic modulus were studied. They found that the mechanical properties of the porous media were related to the pore size distribution of the RVE.

Another way to conduct this research is to reconstruct the real porous media $[9,10]$. Song et al. [11] studied the flow patterns of single-phase and two-phase fluids in porous media based on the statistical parameters of the microstructures of the porous cores and reconstructed digital cores. $\mathrm{Li}$ [12] considered the structural factors on the mesoscale of the porous media; the calculation formula of shear strength and fracture pressure based on the porous media is established per the Terzaghi equation. Raoof [13] used the multiscale analysis method to construct the porous media model in the aperture scale. The basic migration law of the fluid in the hole scale, the relationship between the macroscopic scale and microscopic scale, and the law of the pore scale solute transport and the limitation of the macroscopic scale model were analyzed.

Recently, to study the general characteristics (mechanical properties, thermal conductivity, conductivity, permeability, etc.) of porous media, reconstructing the microstructure of the representative volume element by the quartet structure generation set (QSGS) has received much attention. Jin and Zhao [14] constructed the isotropic or anisotropic microstructures by changing the parameters of the QSGS and studied the relationship between the effective thermal conductivity and the microstructure. Shi et al. [15] constructed thermal barrier coatings (TBC) and analyzed the effect of thermal barrier coatings' (TBC) microstructure on the thermal conductivity based on the statistical method. Chen et al. [16] reconstructed the elementary building block (EBB) model of the shale matrix by using the QSGS. The fluid flow in the RVE was simulated by the Boltzmann method, and the effects of the organic content, grain size, and intergranular pores on the permeability of the RVE in clay facies, calcite, pyrite, and organic matter were analyzed.

In summary, several domestic and foreign scholars have conducted extensive research on the RVE characteristics of porous media, but most of them focus on the porous media permeability, conductivity, thermal conductivity, and other characteristics. The studies on mechanical properties of porous media are rare, and there are few studies on the microstructure and macroscopic properties of the porous media. In this paper, the random porous media model was generated by the QSGS, and the pore structure of the RVE was characterized by the porosity and fractal dimension. The effects of the porosity, fractal dimension, and matrix mechanical properties (elastic modulus, Poisson's ratio) on the mechanical properties of the porous media RVE are analyzed by using a numerical simulation.

\section{Construction of the Real Porous Media RVE}

In micromechanics, the macroscopic porous media are composed of multiple consecutive points, and the mesoscopic space associated with the multiple successive points in the macro is called the representative volume element (RVE) [19, 20]. The representative volume element is the link between the macroscopic scale and microscopic scale, and the scale definition of the RVE of different materials and scales has a certain difference.

The selection of the RVE needs to satisfy the scale of the representative volume element being much smaller than the characteristic scale of the structure, and the scale of the representative volume element should be much larger than the average scale of the nonuniform hole. The main subject of this paper is geomorphic porous media. The macro scale spans from meters to kilometers, while the microscopic experimental scale is generally centimeters or decimeters, and the scale of the micro CT scan is generally $\mathrm{mm}$. The size of the pore structure varies from a few micrometers to several tens of micrometers at the micro CT scan scale. Therefore, $2 \mathrm{~mm} \times 2 \mathrm{~mm} \times 2 \mathrm{~mm}$ is selected as the volume scale of the standard element (RVE) per previous research results [21].

The mesoscopic random porous media are often reconstructed by a pore network [22], fractal Sierpinski carpet model [23], and other methods. Wang et al. [17, 18] used the quartet structure generation set (QSGS) per the statistical principle to reconstruct the microstructure of the porous media based on the macroscopic statistical information and analyzed the porous media characteristics. The porous structure is formed by the four-parameter stochastic growth sets, and it is controlled by four parameters: $c_{d}, D_{i}, P, I_{i}^{m}$. The porosity of the RVE is calculated as follows $[17,18]$ :

$$
P=\frac{\Omega^{p}}{\Omega^{p}+\Omega^{s}}=\frac{\Omega^{p}}{\Omega},
$$

where $\Omega^{p}$ represents the area of the pore phase, $\Omega^{s}$ represents the area of the solid phase, $\Omega$ is the total area of the RVE, and $P$ is the porosity of the RVE.

The distribution probability $c_{d}$ is the random distribution probability of the initial solid particles, which controls the fineness of the porous media structure. The smaller the value of $c_{d}$ is, the smaller the solid particle size is, and the larger the calculated fluctuation is within a given size. $I_{i}^{m}$ represents the growth probability of the pore phase along the eight directions. $D_{i}$ is the growth probability of each element in the growth period in eight directions. When forming a two-dimensional planar porous media structure, each pixel element grows in eight directions, as shown in Figure 1. The directions $1,2,3$, and 4 are the four main directions of the solid particles, and 5, 6, 7, and 8 are the four diagonal directions of the solid particle growth.

The size of the porous media RVE from the CT scanning is small, generally in $\mathrm{mm}$ as a unit for characterization. Thus, the area of the two-dimensional RVE of the fractal porous 


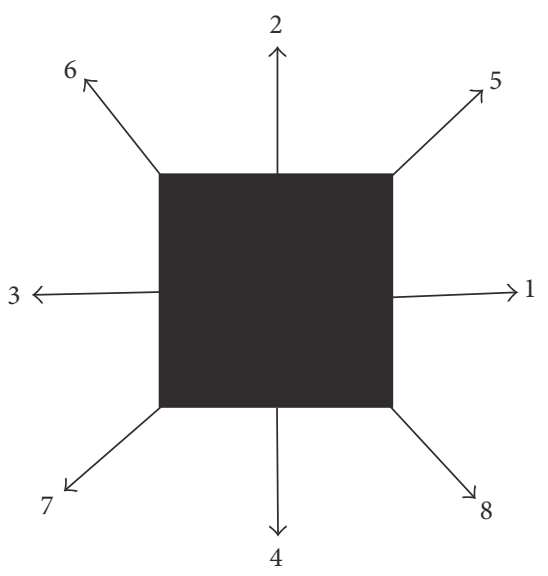

FIGURE 1: The eight growth directions of each point for 2D systems $[17,18]$.

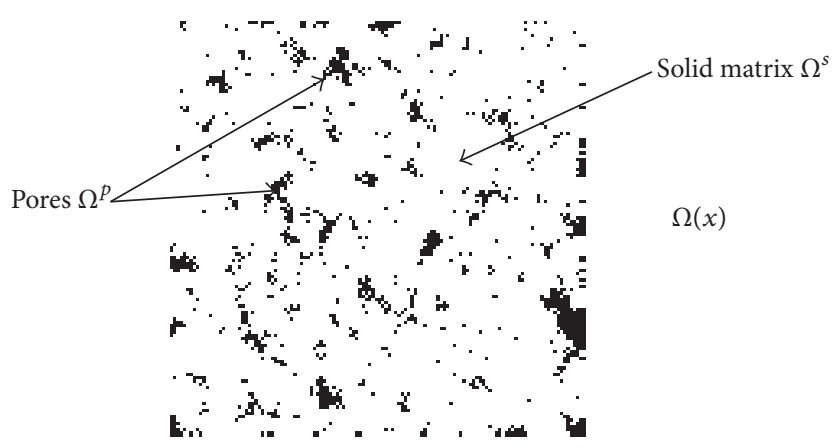

FIGURE 2: The real porous media constructed by the QSGS.

media is set to $2 \mathrm{~mm} \times 2 \mathrm{~mm}$, the number of pixels in the pores and solid particles is 10000 , and the pore structure parameter $d=0.02 \mathrm{~mm}$ of the porous media RVE is far less than the mesoscopic model parameter of $l=2 \mathrm{~mm}$. The mesoscopic model obeys the mesomechanics definition of the RVE containment of enough pore structure features. The parameters of the random porous media generation program are set as follows: the initial solid phase particle probability density is $c_{d}=0.02$, the growth probability of solid particles in eight directions is $D_{1}=D_{2}=D_{3}=D_{4}=D_{5}=D_{6}=D_{7}=$ $D_{8}=0.025$, and $I_{i}^{m}$ is the parameter of the multiphase porous media, which is not defined in the study of the mechanical properties of the two-phase porous media. Figure 2 depicts a random real porous media RVE binary pixel image. In this figure, the white pixel groups represent the pore region and the black pixel groups represent the solid particle region.

The pores size distribution of real porous media obeys the Weibull distribution $[24,25]$. In order to make the models close to the real porous media and easy to converge in the finite element, the QSGS is improved, and the remaining steps are as follows:

(1) The isolated single pores are judged by the four connected domains and eliminated.
(2) The pixel coordinate information is extracted by MATLAB, and it is imported into the finite element program to obtain the finite element model (Figure 3).

\section{Connectivity and Fractal Characterization of Real Porous Media by Reconstruction}

3.1. Connectivity of the Mesoscopic Real Porous Media by Reconstruction. The connectivity problem of porous media is usually based on connected domain detection [24, 25]. A closed pore group is considered as a large pore group, and the pore group contains only an isolated pore or several isolated pores. Since the size of the porous media model reconstructed in this paper is $2 \mathrm{~mm} \times 2 \mathrm{~mm}$, and it contains 10000 pixels, the particle size observed by the microscope is as small as the micron level.

In this paper, the method of four connected domains [26] is used to detect isolated pore groups in a two-dimensional porous media model. The corresponding area scale for the pixel of each isolated pore is $A_{0}=0.02 \mathrm{~mm} \times 0.02 \mathrm{~mm}=$ $20 \mathrm{um} \times 20 \mathrm{um}$.

The pore size is usually calculated with the equivalent maximum circle diameter; therefore, the pore size of $j$ th pore group is

$$
R^{j}=\sqrt{\frac{n \times A_{0}}{\pi}},
$$

where $j$ represents the $j$ th pore group, $n$ is the number of isolated pores contained in the $j$ th pore group, $A_{0}$ is the pore group size that contains a pixel, and $R^{j}$ is the pore size of $j$ th pore group.

Meanwhile, the percentage of pore groups with the same pore size can be expressed as

$$
P_{\mathrm{pz}}=\frac{m}{l},
$$

where $m$ is the number of closed pore groups with the same pore size, $l$ is the number of closed pore groups in a porous media model, and $P_{\mathrm{pz}}$ is the percentage of pore groups with the same pore size.

According to (2) and (3), the pore size distribution characteristics of the reconstructed porous media can be obtained, and the pore size distribution of real porous media models is shown in Figure 4. As is shown in Figure 4, the real porous media models reconstructed by the improved QSGS method are also subject to the Weibull distribution.

3.2. Fractal Characterization of the Mesoscopic Real Porous Media. The microscopic feature of the porosity is a complex problem, and the traditional statistical method can characterize the distribution of pore area, shape, and radius. However, the spatial position of the different porous media porosity groups has no specific parameters for the characterization and analysis. To describe this type of complex structure, Mandelbrot proposed a fractal theory that provides a direction for the study of the complex phenomena. Meanwhile, the experimental research of foreign scholars has proved the 


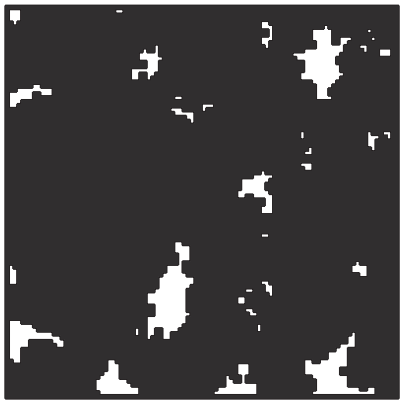

$P=0.08$

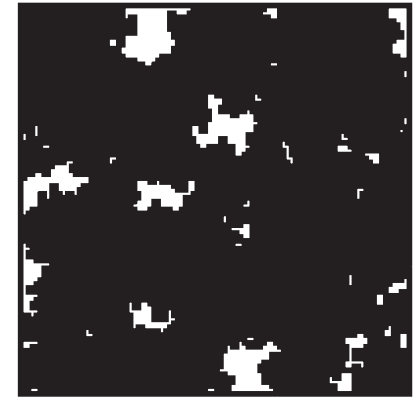

$P=0.09$

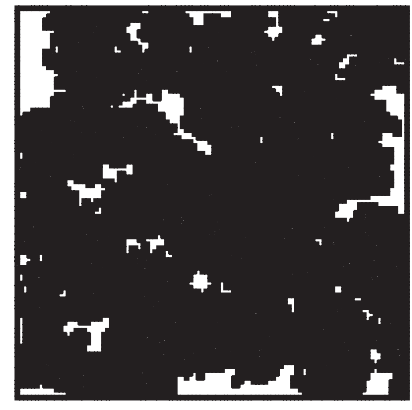

$P=0.10$

FIGURE 3: The real porous media model reconstructed by the improved QSGS.
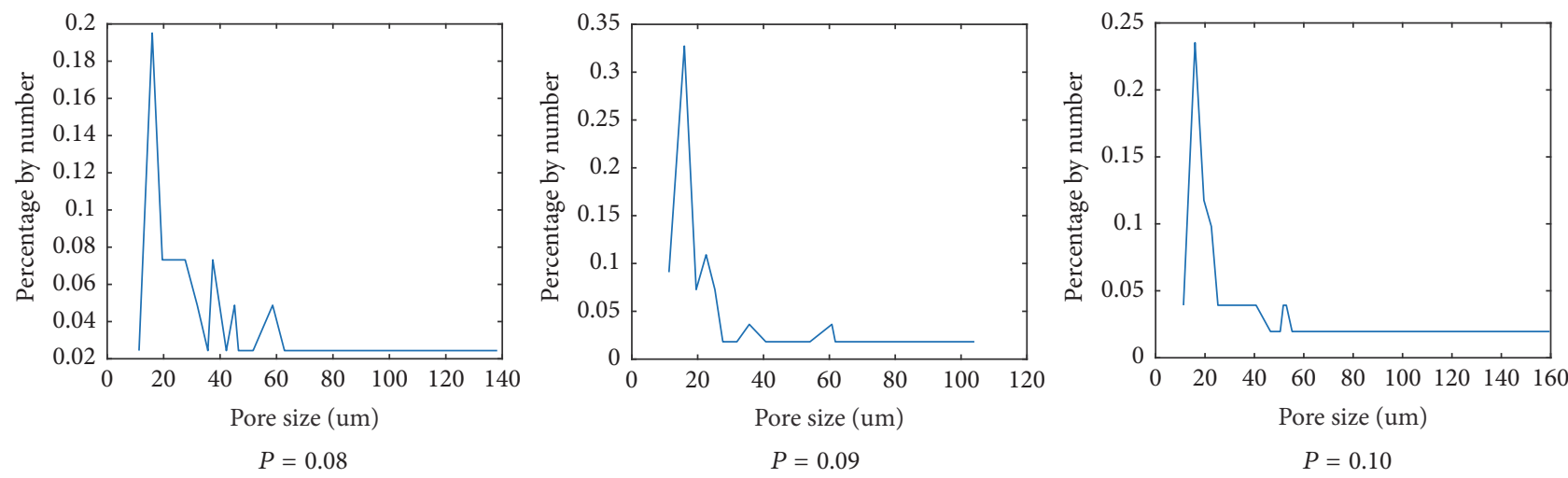

FIGURE 4: Distribution of 2D pore sizes in reconstructed porous media with different porosities.

feasibility of using fractal method to study real porous media $[27,28]$

For the two-dimensional stochastic porous media, the fractal dimension of the porosity reflects the change in the pore structure characteristics to a certain extent. The fractal dimension can be calculated by the box-counting method $[7,29]$ :

$$
D=-\lim _{r \rightarrow 0} \frac{\lg N(r)}{\lg r},
$$

where $D$ represents the fractal dimension of the porous media, and the fractal body in the three-dimensional case follows the interface convention: $D_{3-\mathrm{D}}=D_{2-\mathrm{D}}+1=D_{1-\mathrm{D}}+2$, $D_{3-\mathrm{D}}, D_{2-\mathrm{D}}, D_{1-\mathrm{D}}$ which represents the fractal dimension of the fractal body observed from space, cross section, and stub, respectively. $r$ is the size of the box, and $N(r)$ is the number of boxes that cover the porous media. For example, we divide the entire RVE region into a number of boxes of size $r$, then some boxes contain pores, and some boxes do not contain pores. $N(r)$ is the number of boxes that contain pores.

\section{Numerical Simulation and Results of the Analysis}

4.1. Model Hypothesis. To realize the feasibility of the numerical simulation, the following assumptions are made for the model:
(1) In consideration of the pore structure of the real porous media model by reconstruction, the pore structure was characterized by the porosity and pore fractal dimension.

(2) It is assumed that the matrix is uniform elastoplastic and that the stress-strain of the matrix is always in the elastic-plastic phase during the whole loading process, only the overall effects of RVE are considered, regardless of the local structural failure during the whole loading process.

(3) The effect of the air pressure on the pore deformation in the porous media is ignored.

(4) The bond changes, particle swings, and other effects of the inner matrix of the RVE are ignored.

(5) During the loading process, the friction on different surfaces is ignored.

4.2. Constitutive Model of Matrix. The regional scale is much larger than the observed pore size by the microscope. Therefore, it can be assumed that the solid phase matrix of the stochastic pore model is a continuous homogeneous medium composed of elastoplastic materials $[30,31]$. 


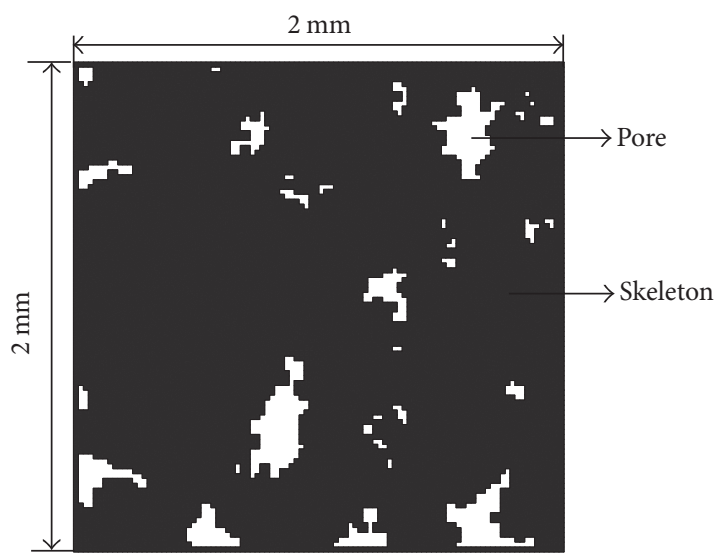

(a) Model diagram

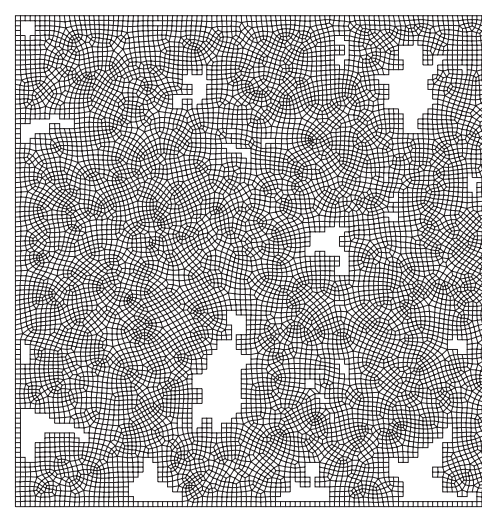

(b) FEM meshing

FIGURE 5: The model of the finite element.

Currently, according to the effective stress principle and the elastic equilibrium differential equation, the elastic equilibrium differential equation of the matrix is

$$
\sigma_{i j, j}^{\prime}+f_{i}=0
$$

where $f_{i}$ denotes force components and $\sigma_{i j, j}^{\prime}$ represents the stress components.

In the process of compression, the continuity equation of matrix deformation can be expressed as

$$
\varepsilon_{i j}=\frac{1}{2}\left(u_{i, j}+u_{j, i}\right),
$$

where $\varepsilon_{i j}$ represents strain tensor and $u$ is displacement.

The constitutive relation of the matrix is an elastoplastic model, and its incremental form can be expressed as

$$
\left\{d \sigma_{i j}^{\prime}\right\}=\left[D_{\mathrm{ep}}\right]\left\{d \varepsilon_{i j}\right\},
$$

where $\left[D_{\mathrm{ep}}\right]$ is elastoplastic matrix.

The Mises yield criterion for the plastic yield is

$$
f=J_{2}-k^{2}=0,
$$

where $k$ represents the material parameters and $J_{2}$ is the second invariant of the stress bias.

The second invariant of the stress bias expression is

$$
J_{2}=\frac{1}{3}\left[\left(\sigma_{1}-\sigma_{2}\right)^{2}+\left(\sigma_{2}-\sigma_{3}\right)^{2}+\left(\sigma_{3}-\sigma_{1}\right)^{2}\right],
$$

where $\sigma_{1}, \sigma_{2}, \sigma_{3}$ represent three principal stresses of matrix, in two-dimensional space, $\sigma_{3}=0$.

4.3. Description of the Model. The regional scale is much larger than the observed pore size by the microscope. The porosity range of the real porous media is 0.06 to 0.25 , and 25 two-dimensional porous media were randomly generated by the improved QSGS in MATLAB. There are nine models with a porosity of 0.08 , nine models with a porosity of 0.09 , and seven models with a porosity of 0.1 to 0.16 . In this paper, the transverse comparisons have the same porosity and different fractal dimension, and the vertical comparisons have a different porosity and fractal dimension.

For the output pore pixel coordinates in MATLAB, the two-dimensional porous media geometric model data is imported into ABAQUS via the python script. The model is meshed by global partition and a structured grid technology. The pixel size is $0.02 \mathrm{~mm} \times 0.02 \mathrm{~mm}$, the global mesh size is set to 0.02 , and the result of the meshing is shown in Figure 5.

4.4. The Set Initial Conditions and Boundary Conditions. When a porous medium is loaded into a microscopic physical experiment, the longitudinal deformation of the upper boundary is consistent. Therefore, using a rigid body loading in the upper part of the model, the longitudinal displacement of the lower boundary is constrained. When the numerical simulation experiment is carried out, the rigid body is loaded into the upper part of the model and the lower boundary restrains its longitudinal displacement. For the above random porous media ballast simulation, the longitudinal total set variable is set as $0.04 \mathrm{~mm}$, the longitudinal total strain is $2 \%$ after the completion of the simulation, and the loading rate is set as $4 \times 10^{-5} \mathrm{~mm}$ per increment to make the results easier to converge. The remaining initial parameters are set as follows:

(1) Elastic parameters are as follows: $E=21 \mathrm{GPa}, v=0.3$, and $\sigma$ is the yield stress.

(2) The matrix mechanical parameters are shown in Table 1.

\subsection{Analysis of Fractal Porous Media}

4.5.1. Mechanical Properties of the Fractal Porous Media with the Same Porosity. To study the mechanical properties of different fractal porous media under the same matrix mechanical properties, the fractal porous media models with a porosity of 0.08 and a porosity of 0.09 were selected. The fractal dimension was calculated by MATLAB, and twelve 


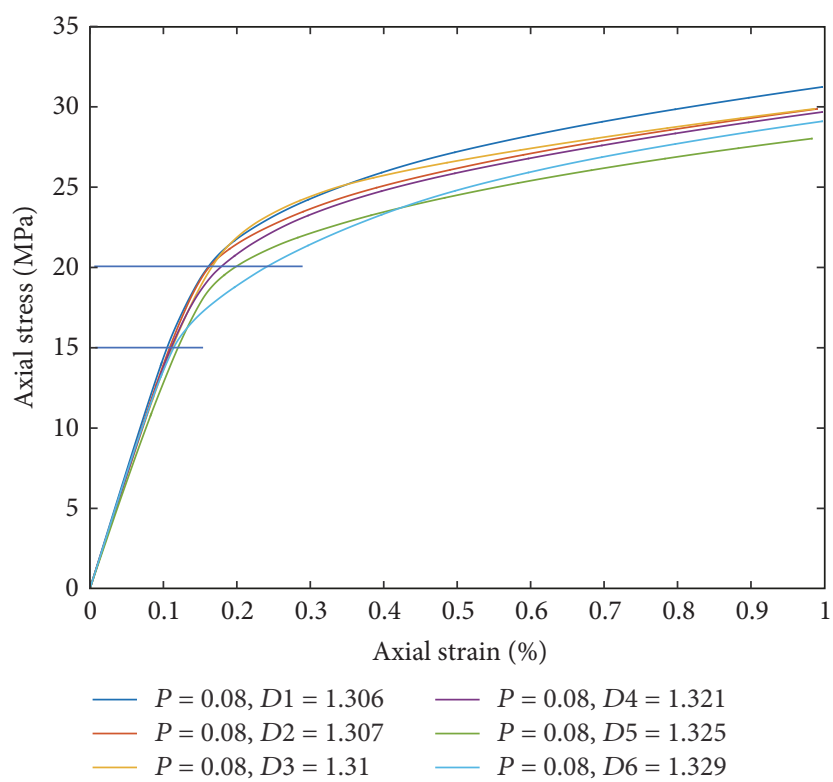

(a) $P=0.08$

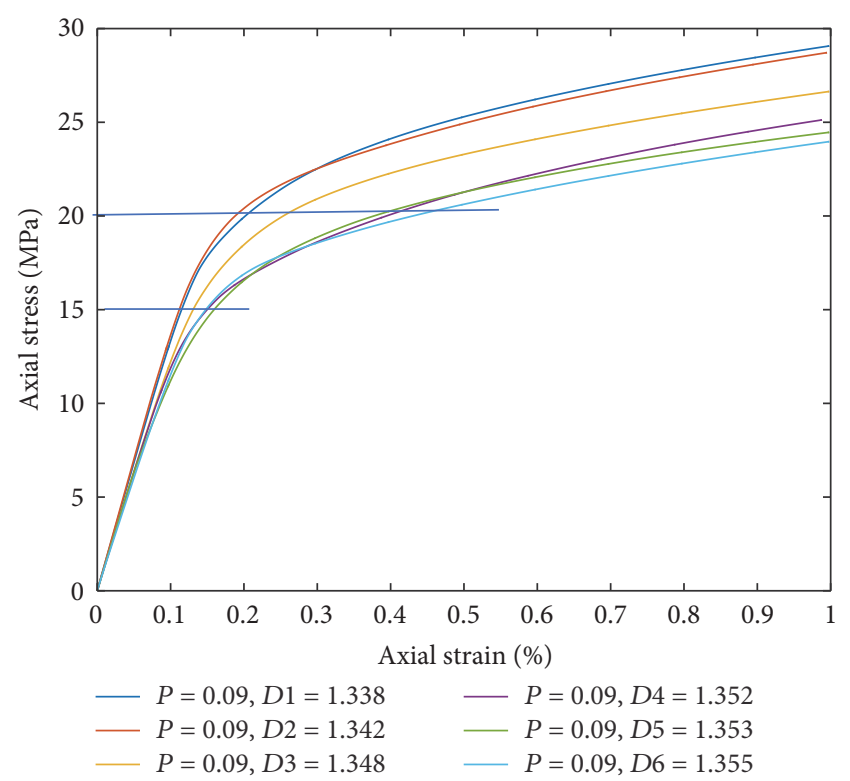

(b) $P=0.09$

FIGURE 6: The stress-strain curve of the different fractal dimensions under the same porosity.

TABLE 1: The matrix mechanical parameters.

\begin{tabular}{lcccc}
\hline$\sigma(\mathrm{MPa})$ & 20.7 & 21.7 & 22.4 & 23.8 \\
\hline$\varepsilon_{\text {eq }}$ & 0 & 0.002 & 0.004 & 0.0098 \\
\hline
\end{tabular}

TABLE 2: The porosity and fractal dimension of the RVEs.

\begin{tabular}{lcccccc}
\hline \multirow{2}{*}{ Porosity } & \multicolumn{6}{c}{ Number } \\
& 1 & 2 & 3 & 4 & 5 & 6 \\
\hline 0.08 & 1.306 & 1.307 & 1.31 & 1.321 & 1.325 & 1.329 \\
0.09 & 1.338 & 1.342 & 1.348 & 1.352 & 1.353 & 1.355 \\
\hline
\end{tabular}

models with different fractal dimensions and porosity were selected for simulation. The porosity and fractal dimension of the porous media are shown in Table 2 . The RVE mechanical characteristics are shown in Figure 6.

Figures 6(a) and 6(b) depict the stress-strain curves of the RVEs of porous media with different fractal dimensions when the porosity of the porous media is 0.08 and 0.09 . It shows that the mechanical properties of the porous medium RVE will decrease with the increase of the pore fractal dimension under the same porosity, and this trend has some fluctuation. The fractal dimension can be used to describe complex structures, but for the inclusion of geometric, spatial, and other information about the complex structure of the pores, the fractal dimension is not a perfect representation of the parameters. The stress-strain curves of the porous media with different fractal dimensions at $P=0.08$ and $P=0.09$ were identified at pressures of $15 \mathrm{MPa}$ and $20 \mathrm{MPa}$, respectively. When the pressure is $15 \mathrm{MPa}, P=0.08$ corresponds to different fractal dimensions. The stress-strain curve of the porous medium RVE is basically in the linear elastic phase, and $P=0.09$ corresponds to different fractal dimensions. The yield strength of the porous media RVE decreases with the increase of the porosity in the case of the same mechanical properties as the matrix. When the pressure is $20 \mathrm{MPa}, P=0.08$ corresponds to a different fractal dimension porous media RVE stress and strain curve, which is slightly within the yield stage, and $P=0.09$ corresponds to different fractal dimension porous media in which the RVE stress and strain curves have all entered the yield stage. Meanwhile, the lithological variation of the porous media RVE is inferred from the fractal dimension when the difference in the fractal dimension is large with the same porosity, and the elastic modulus and yield strength of the porous media RVE decrease with the increase of the fractal dimension, which indicates that the fractal dimension is more accurate than the mechanical properties of the porous media RVE characterized by the porosity.

Based on the above analysis, the RVE mechanical properties (elastic modulus and yield strength) decrease with the increase of the pore fractal dimension of the porous media in the case of the same lithology and porosity of the real porous media. At the same time, the fractal dimension is more accurate than the porosity when studying the pore characteristics of the porous media. However, the trend has a certain fluctuation because the fractal dimension is still insufficient to fully characterize the spatial and geometric characteristics of the pore structure in the RVE of porous media.

4.5.2. Mechanical Properties of Fractal Porous Media with Different Porosity. To further study the effect of the pore fractal of the porous media on the lithological characteristics of porosity, a porous medium with a porosity of $0.1 \sim 0.16$ was formed by setting different initial porosity parameters. The fractal dimension of the porous media not only is related to 
TABLE 3: The porosity and fractal dimension of the RVEs.

\begin{tabular}{lccccc}
\hline \multirow{2}{*}{ Parameters } & \multicolumn{5}{c}{ Number } \\
& 1 & 2 & 3 & 4 & 5 \\
\hline Porosity & 0.08 & 0.09 & 0.10 & 0.11 & 0.16 \\
Fractal dimension & 1.306 & 1.338 & 1.369 & 1.403 & 1.484 \\
\hline
\end{tabular}

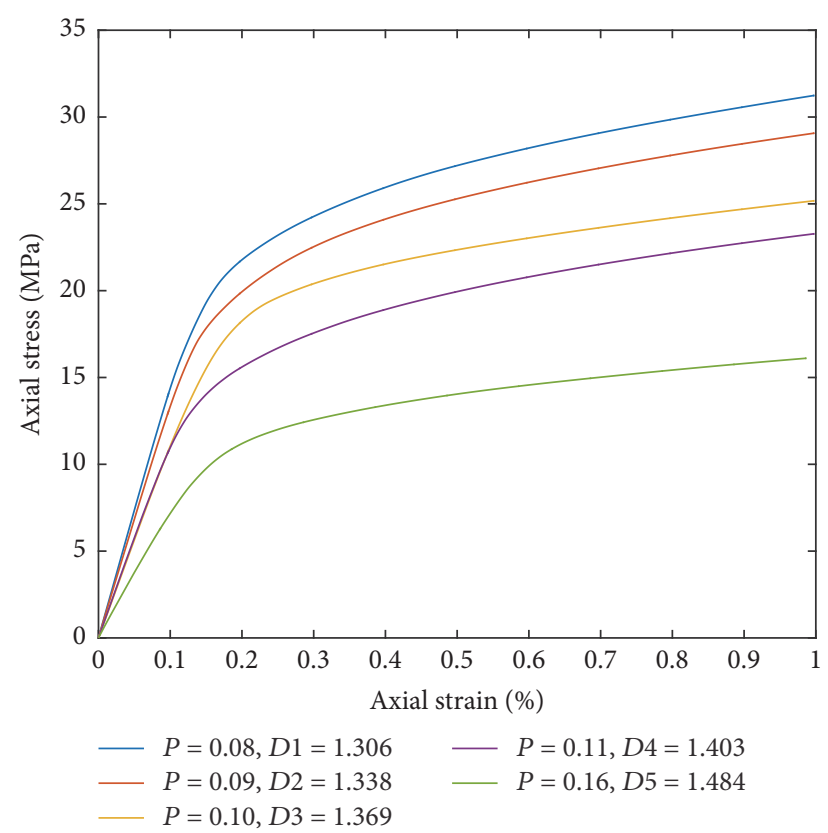

FIgURE 7: The stress-strain curves at different porosities when the fractal dimension difference is large.

the porosity, but also has some relationship with the pore structure. To study the effect of the fractal dimension of the porous media on its RVE mechanical characteristics, several models with a large difference in the fractal dimension are selected, and the results were compared and analyzed. The initial pore and fractal characteristics of the random fractal porous media are shown in Table 3.

Figure 7 depicts the stress-strain curves of the porous media RVE when the porosity and the pore fractal dimension of the porous media RVE are different. By comparing the stress-strain curves of $P=0.08, D 1=1.306, P=0.09, D 2=$ 1.338, $P=0.10, D 3=1.369, P=0.11, D 4=1.403$, and $P=$ $0.16, D 5=1.484$, the elastic-plastic mechanical properties (elastic modulus and yield strength) of the porous media show a decreasing trend with the increase of the porosity and fractal dimension when the elastic-plastic mechanical properties of the matrix are consistent. At the same time, the fractal dimension of the pore is positively correlated with the increase of the porosity of the porous media RVE, and this kind of correlation is also fluctuating. By comparing the stress-strain curve of $P=0.10, D 3=1.369$ and $P=0.11$, $D 4=1.403$, it can be found that the trend of the elastic modulus shows a fluctuation, but the yield strength of the porous media RVE at $D 4=1.403$ is obviously lower than the yield strength of the porous media RVE at $D 3=1.369$.

In conclusion, the pore fractal dimension of the porous medium RVE has a positive correlation with the porosity of the porous media. Moreover, the mechanical properties (elastic modulus, yield strength) of the porous media RVE decrease with the increase of the porosity and fractal dimension of the porous media when the matrix mechanical properties of the porous media are consistent.

4.5.3. Mechanical Properties of the Fractal Porous Media with Different Matrix of Different Mechanical Properties. The mechanical properties of the porous media RVE are related not only to the porosity and fractal dimension of the porous media but also to the matrix mechanical properties of the RVE. During the course of loosening of the matrix from loose to dense, the mechanical properties of the RVE are changed due to the change in the particle size and particle cohesiveness at the smaller scale of the matrix. For a further demonstration of this experiment, the two microscopic porous media models with $P=0.08, D=1.306$ and $P=0.16, D=1.484$ were selected. The mechanical properties of the mesoscopic porous media RVE are discussed in the case of the porosity and pore fractal dimension.

(1) Effects of the Matrix Elastic Modulus on the Mechanical Properties of the Porous Media. The two microporous media with $P=0.08, D=1.306$ and $P=0.16, D=1.484$ were selected for the simulation, and the matrix elastic modulus was set as $21 \mathrm{GPa}, 24 \mathrm{GPa}, 26 \mathrm{GPa}, 28 \mathrm{GPa}$, and $30 \mathrm{GPa}$. The remaining parameters remain unchanged, and the numerical simulation results are shown in Figures $8(a)$ and $8(b)$.

Figures $8(\mathrm{a})$ and $8(\mathrm{~b})$ depict the stress-strain curves of the porous media RVE with the porosity $P=0.08$ and the fractal dimension $D=1.306$ and with the porosity $P=0.16$ and the fractal dimension $D=1.484$, respectively. It can be seen from Figures $8(a)$ and $8(\mathrm{~b})$ that when the porosity and fractal dimension of the fractal porous media are consistent, the stress-strain curve is surrounded by a higher stress-strain curve of the matrix elastic modulus as matrix elastic modulus decreases. It can be explained by the mechanical properties (elastic modulus, yield strength) of the porous medium RVE being consistent with the matrix elastic modulus of the porous media when the porosity, fractal dimension of the porous media RVE, and Poisson's ratio of the porous media matrix are the same in the fractal porous media RVE elastoplastic deformation stage. Then, the stress-strain curves of the porous media with a different initial elastic modulus are close to each other, which indicates that the deformation of the RVE of porous media increases with the constant pressure of the porous media RVE. The inner pores are gradually compacted, the stress distribution caused by the pore structure distribution is uneven, and the stress concentration phenomenon is decreasing. The stress in each region of the RVE gradually reaches a macroscopic equilibrium, the regionalization of the force, and the displacement change is stratified. The pore deformation ratio is gradually reduced, the pore deformation and the matrix deformation gradually reach the macroscopic equilibrium, and the macro balance 


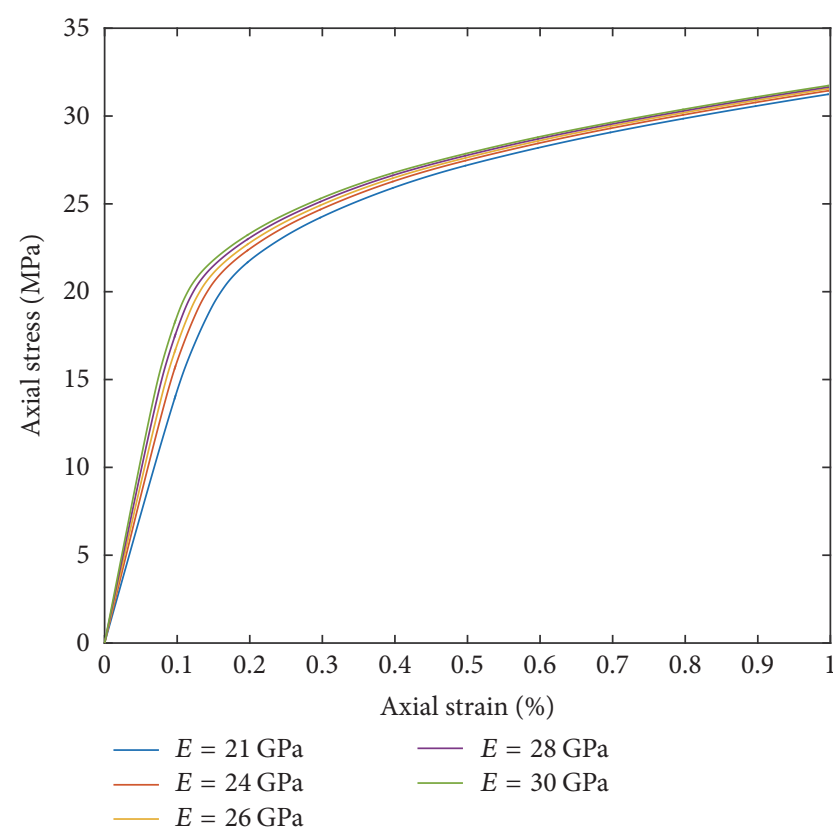

(a) $P=0.08, D=1.306$

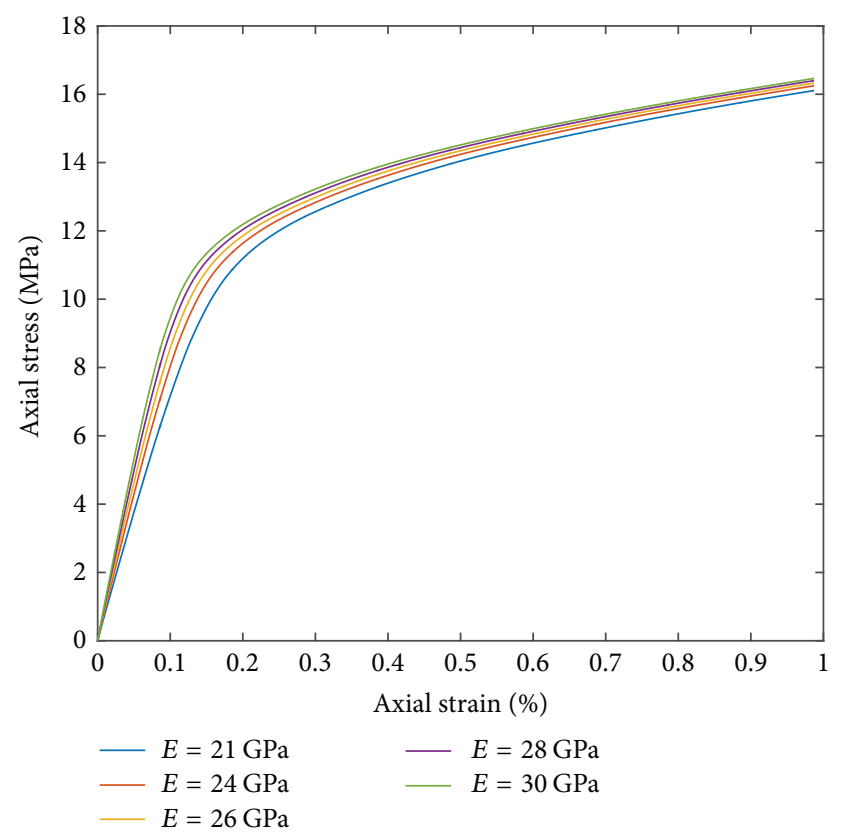

(b) $P=0.16, D=1.484$

FIGURE 8: The stress and strain curves with the porosity and fractal dimension consistent with the variation of the elastic modulus of the matrix.

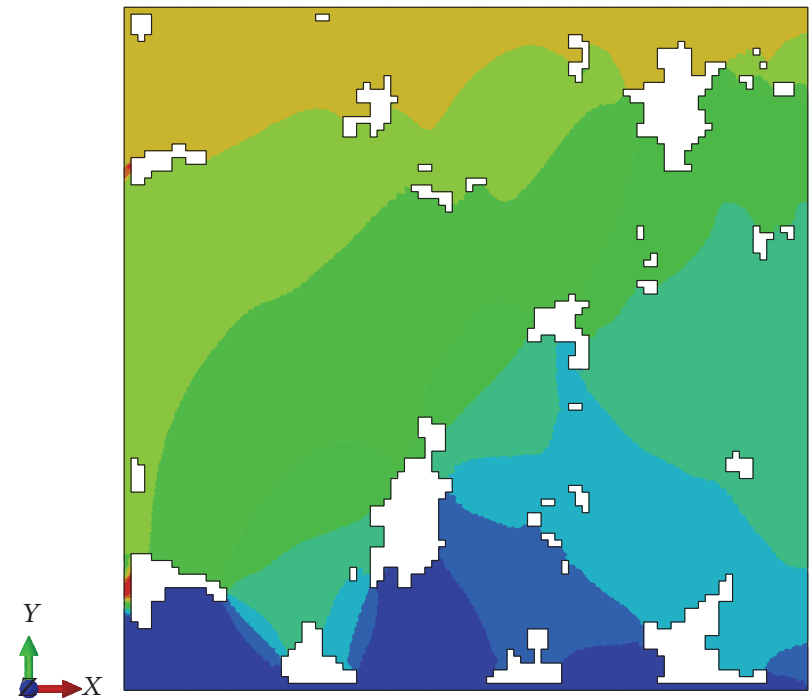

(a)

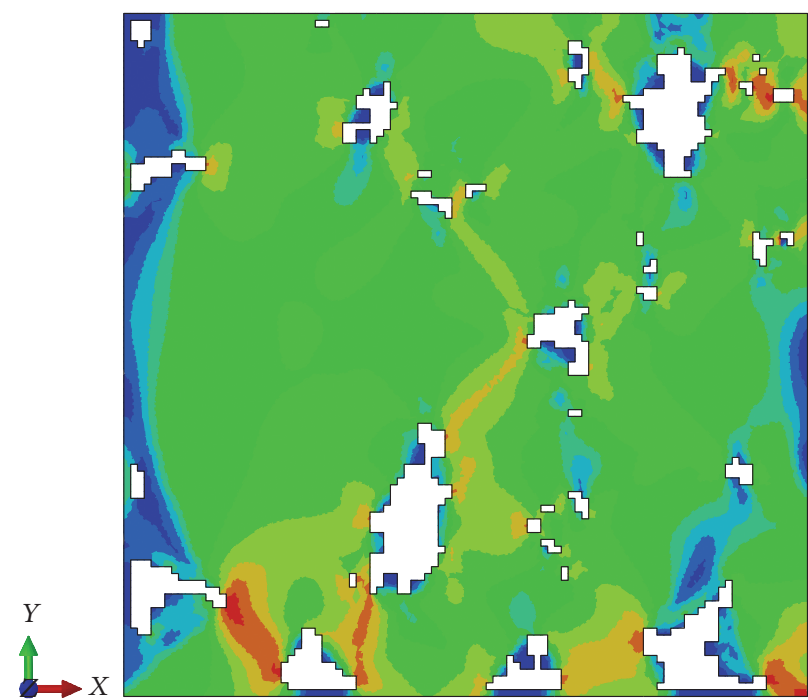

(b)

FIgURE 9: The RVE diagram ((a) depicts the displacement contour and (b) depicts the stress contour).

of the force region and the failure of the RVE are not formed after the infinite compaction of the void (Figure 9).

(2) Effects of the Matrix Poisson's Ratio on Mechanical Properties of the Porous Media. The RVE of the two microscopic porous media with $P=0.08, D=1.306$ and $P=0.16$, $D=1.484$ were selected. The range of Poisson's ratio of the porous rock is generally from 0.21 to 0.35 . Thus, Poisson's ratios of the matrix are set as $0.26,0.27,0.28$, and 0.29 , and the remaining parameters remain unchanged. The numerical simulation results are as follows.

Figures 10(a) and 10(b) depict the stress-strain curves of the porous media RVE with $P=0.08, D=1.306$ and $P=0.16, D=1.484$, respectively. The stress-strain curve is basically the same when the matrix Poisson's ratio is $0.26,0.27,0.28$, and 0.29 , and it can be deduced that the macroscopic mechanical properties of the porous media RVE in the elastic-plastic deformation stage are not correlated with Poisson's ratio of the porous media matrix when the 


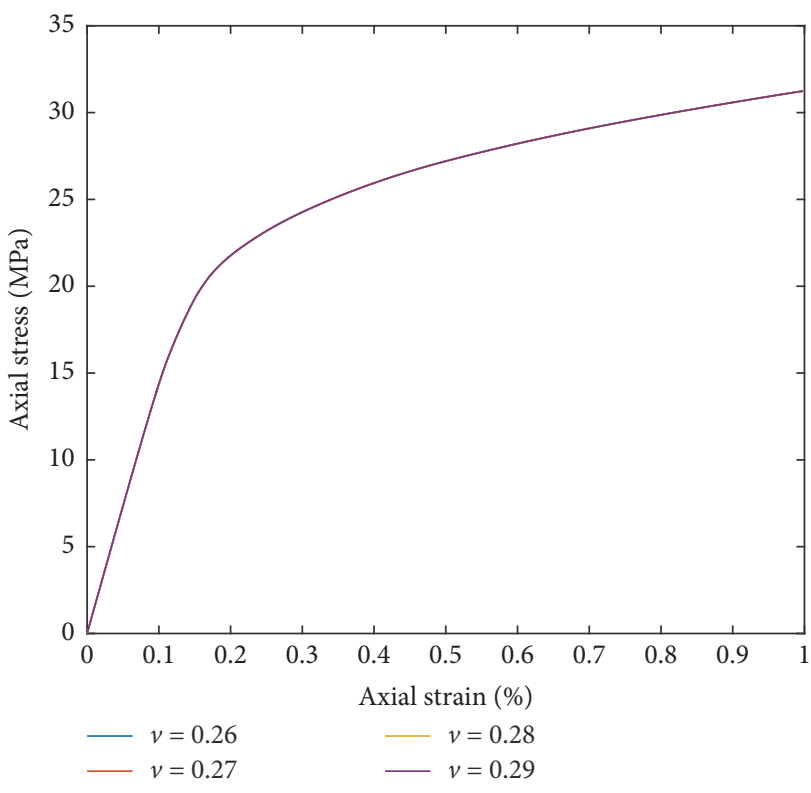

(a) $P=0.08, D=1.306$

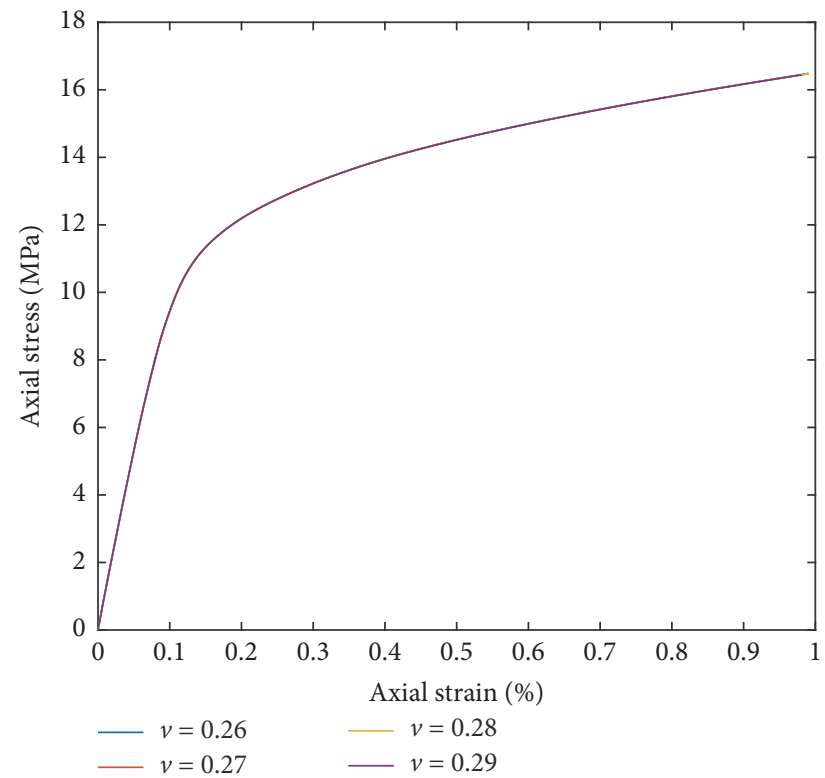

(b) $P=0.16, D=1.484$

Figure 10: The stress and strain curves with the porosity and fractal dimension consistent with the variation of Poisson's ratio of the matrix.

porosity, fractal dimension, plasticity, and elastic modulus of the porous medium RVE are consistent. In a certain range, the influence of the matrix Poisson's ratio on its RVE mechanical properties can be neglected.

\section{Conclusion and Analysis}

In this paper, the QSGS is improved, and the connectivity of the reconstructed porous media models are analyzed. The relationship between the microstructure characteristics of the RVE and the RVE mechanical properties of the fractal porous media is analyzed by numerical simulation.

(1) The QSGS is improved, and the porous media model is reconstructed by improved QSGS. The reconstructed porous media model is consistent with the statistical distribution of real pores (Weibull distribution). Meanwhile, these models can be directly introduced into the finite element and calculated.

(2) The macroscopic mechanical properties (elastic modulus, yield strength) of the RVE decrease with the increase of the porosity and fractal dimension, and the trend gradually becomes more obvious with the increase in the difference between the porosity and fractal dimension.

(3) When the porosity and the matrix lithologic characteristics are consistent, the mechanical characteristics of porous media are related to the fractal dimension.

(4) At present, porosity is used as a parameter for micromechanics homogenization of mechanical properties of porous media. In fact, it is more accurate to deduce the homogenization equation of pore fractal dimension for real porous media, such as reservoir core.

\section{Conflicts of Interest}

The authors declare that there are no conflicts of interest regarding the publication of this paper.

\section{Acknowledgments}

This research is supported by National Science and Technology Major Project of China under Grant nos. 2017ZX05037001 and 2017ZX05013001.

\section{References}

[1] F. Golfier, M. Quintard, B. Bazin, and R. Lenormand, "Corescale description of porous media dissolution during acid injection-part II: calculation of the effective properties," Computational and Applied Mathematics, vol. 25, no. 1, pp. 406-413, 2006.

[2] O. Prokopiev, Mechanical Properties of Porous Solids [M.S. thesis], 2009.

[3] G. Lu, G. Q. Lu, and Z. M. Xiao, "Mechanical properties of porous materials," Journal of Porous Materials, vol. 6, no. 4, pp. 359-368, 1999.

[4] I. Yakub, J. Du, and W. O. Soboyejo, "Mechanical properties, modeling and design of porous clay ceramics," Materials Science and Engineering: A Structural Materials: Properties, Microstructure and Processing, vol. 558, pp. 21-29, 2012.

[5] K. Zhao, Y.-F. Tang, Y.-S. Qin, and J.-Q. Wei, "Porous hydroxyapatite ceramics by ice templating: freezing characteristics and mechanical properties," Ceramics International, vol. 37, no. 2, pp. 635-639, 2011.

[6] X. Wang, Z. Yao, J. Ma, and Y. Pan, "Analysis of void characteristics in shear band of porous media (2): maximum void ratio," Chinese Journal of Rock Mechanics and Engineering, vol. 23, no. 15, pp. 2519-2522, 2004. 
[7] B. B. Mandelbrot, The Fractal Geometry of Nature, W. H. Freeman, New York, NY, USA, 1983.

[8] G. Pia, L. Casnedi, M. Ionta, and U. Sanna, "On the elastic deformation properties of porous ceramic materials obtained by pore-forming agent method," Ceramics International, vol. 41, no. 9, pp. 11097-11105, 2015.

[9] V.-H. Nguyen, E. Rohan, and S. Naili, "Multiscale simulation of acoustic waves in homogenized heterogeneous porous media with low and high permeability contrasts," International Journal of Engineering Science, vol. 101, pp. 92-109, 2016.

[10] T. G. Zieliński, "Generation of random microstructures and prediction of sound velocity and absorption for open foams with spherical pores," The Journal of the Acoustical Society of America, vol. 137, no. 4, pp. 1790-1801, 2015.

[11] R. Song, J. Liu, and D. Qin, "Numerical simulation of two phase flow in reconstructed pore network based on lattice boltzmann method," International Journal of Computer Science Issues, vol. 10, no. 3, pp. 193-200, 2013.

[12] P. Li, "Mathematical models of flow-deformation coupling for porous media," Chinese Journal of Rock Mechanics and Engineering, vol. 23, no. 16, p. 2842, 2004 (Chinese).

[13] A. Raoof, Reactive/Adsorptive transport in (partially-) saturated porous media: from pore scale to core scale [Ph.D. thesis], 2011.

[14] X. Q. Jin and C. Y. Zhao, "Numerical investigation on the effective thermal conductivity of plasma sprayed zirconia coatings," Ceramics International, vol. 41, no. 10, pp. 14915-14923, 2015.

[15] H. Shi, C. Y. Zhao, and B. X. Wang, "Modeling the thermal radiation properties of thermal barrier coatings based on a random generation algorithm," Ceramics International, vol. 42, no. 8, pp. 9752-9761, 2016.

[16] L. Chen, Q. Kang, Z. Dai, H. S. Viswanathan, and W. Tao, "Permeability prediction of shale matrix reconstructed using the elementary building block model," Fuel, vol. 160, article no. 9475, pp. 346-356, 2015.

[17] M. Wang, N. Pan, J. Wang, and S. Chen, "Mesoscopic simulations of phase distribution effects on the effective thermal conductivity of microgranular porous media," Journal of Colloid and Interface Science, vol. 311, no. 2, pp. 562-570, 2007.

[18] M. Wang, J. Wang, N. Pan, and S. Chen, "Mesoscopic predictions of the effective thermal conductivity for microscale random porous media," Physical Review E: Statistical, Nonlinear, and Soft Matter Physics, vol. 75, no. 3, Article ID 036702, 2007.

[19] J. Zhou, A. M. Gokhale, A. Gurumurthy, and S. P. Bhat, "Realistic microstructural RVE-based simulations of stressstrain behavior of a dual-phase steel having high martensite volume fraction," Materials Science and Engineering: A Structural Materials: Properties, Microstructure and Processing, vol. 630, pp. 107-115, 2015.

[20] K. Yazdchi, S. Srivastava, and S. Luding, "Microstructural effects on the permeability of periodic fibrous porous media," International Journal of Multiphase Flow, vol. 37, no. 8, pp. 956966, 2011.

[21] A. Q. Raeini, M. J. Blunt, and B. Bijeljic, "Direct simulations of two-phase flow on micro-CT images of porous media and upscaling of pore-scale forces," Advances in Water Resources, vol. 74, pp. 116-126, 2014.

[22] M. Hekmatzadeh, M. Dadvar, and M. Sahimi, "Pore-network simulation of unstable miscible displacements in porous media," Transport in Porous Media, vol. 113, no. 3, pp. 511-529, 2016.
[23] P. M. Adler, "Fractal porous media III: transversal stokes flow through random and sierpinski carpets," Transport in Porous Media, vol. 3, no. 2, pp. 185-198, 1988.

[24] Z. Jiang, Characterisation of pore sizes and connectivity in 3D porous media, 2006, http://proceedings.dtu.dk/fedora/ repository/dtu:1696/OBJ/article.pdf.

[25] H. Shen, S. M. Oppenheimer, D. C. Dunand, and L. C. Brinson, "Numerical modeling of pore size and distribution in foamed titanium," Mechanics of Materials, vol. 38, no. 8-10, pp. 933-944, 2006.

[26] L. Sun, X. Wang, X. Jin, J. Li, and S. Wu, “Three dimensional characterization and quantitative connectivity analysis of micro/nano pore space," Petroleum Exploration and Development, vol. 43, no. 3, pp. 490-498, 2016.

[27] A. H. Thompson, "Fractals in rock physics," Annual review of Earth and planetary sciences, 19, pp. 237-262, 1991.

[28] P.-Z. Wong, "The statistical physics of sedimentary rock," Physics Today, vol. 41, no. 12, pp. 24-32, 1988.

[29] A. Dathe, S. Eins, J. Niemeyer, and G. Gerold, "The surface fractal dimension of the soil-pore interface as measured by image analysis," Geoderma, vol. 103, no. 1-2, pp. 203-229, 2001.

[30] W. Q. Shen and J. F. Shao, "Some micromechanical models of elastoplastic behaviors of porous geomaterials," Journal of Rock Mechanics and Geotechnical Engineering, vol. 9, no. 1, pp. 1-17, 2017.

[31] F. Gruttmann, R. Sauer, and W. Wagner, “Theory and numerics of three-dimensional beams with elastoplastic material behaviour," International Journal for Numerical Methods in Engineering, vol. 48, no. 12, pp. 1675-1702, 2000. 

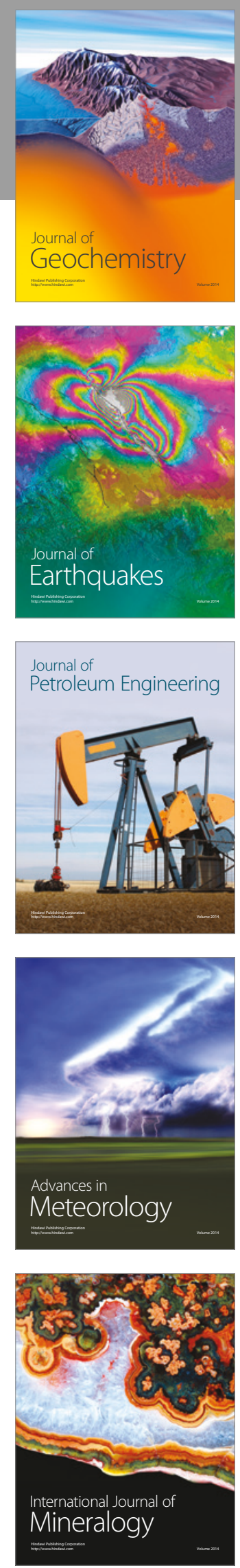
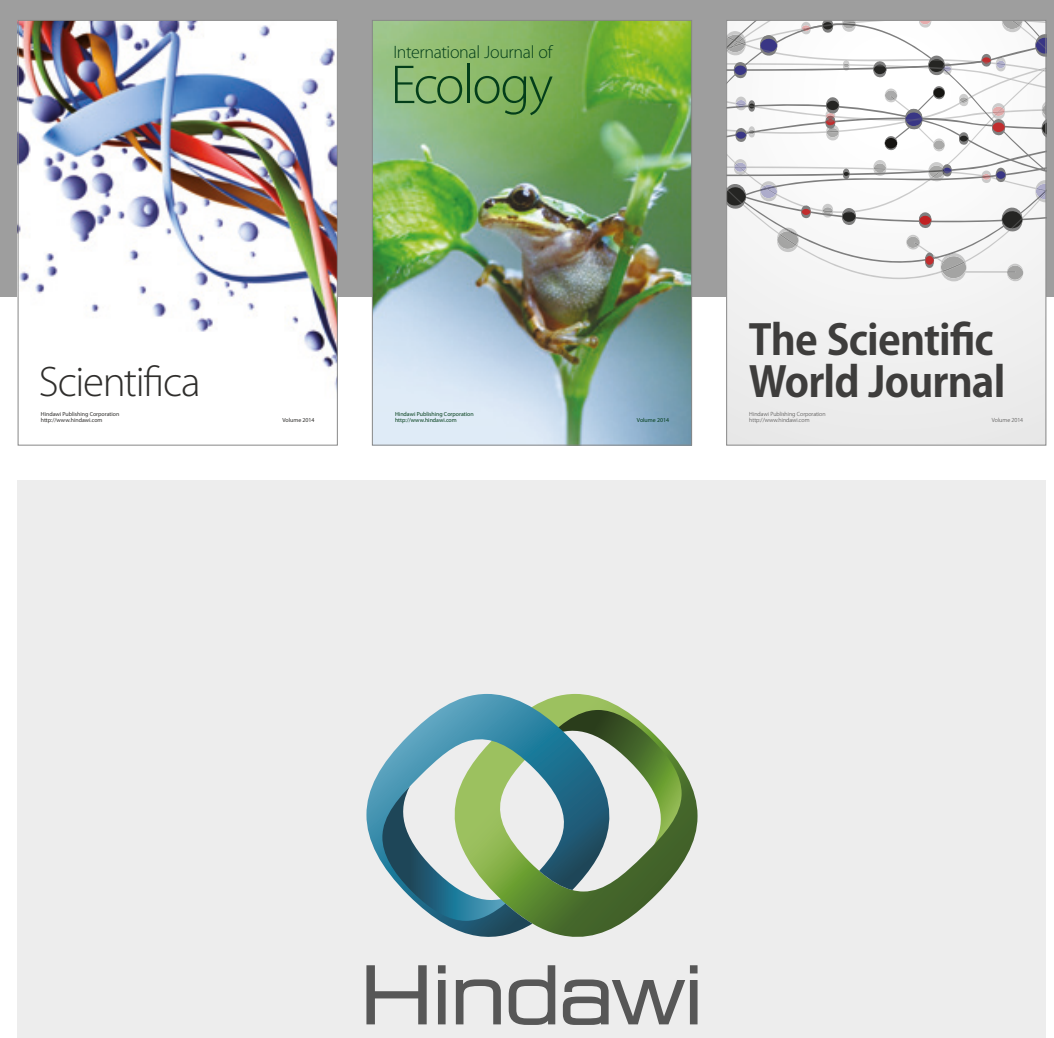

Submit your manuscripts at

https://www.hindawi.com
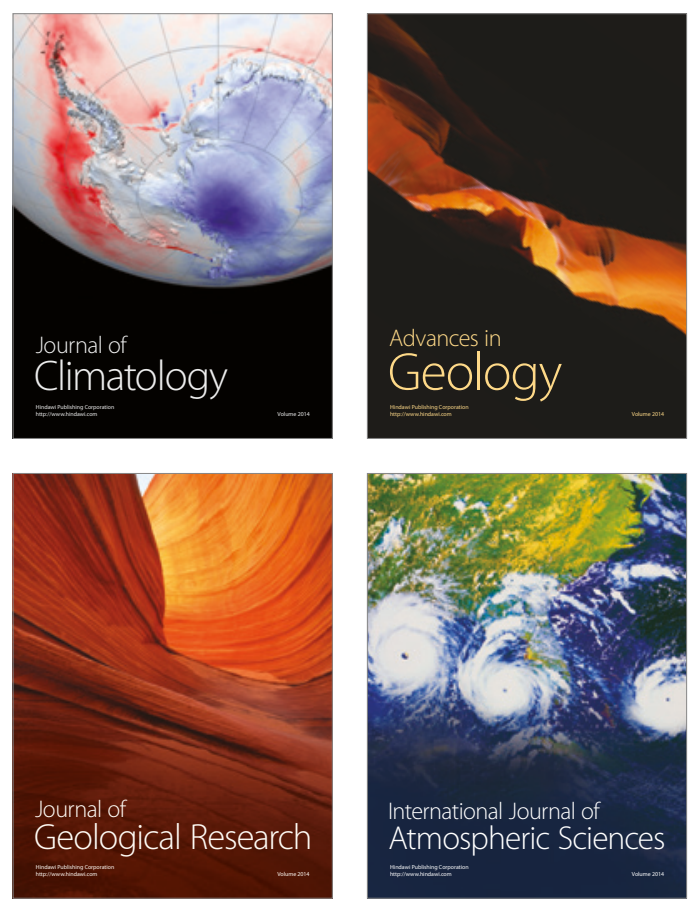

The Scientific

World Journal
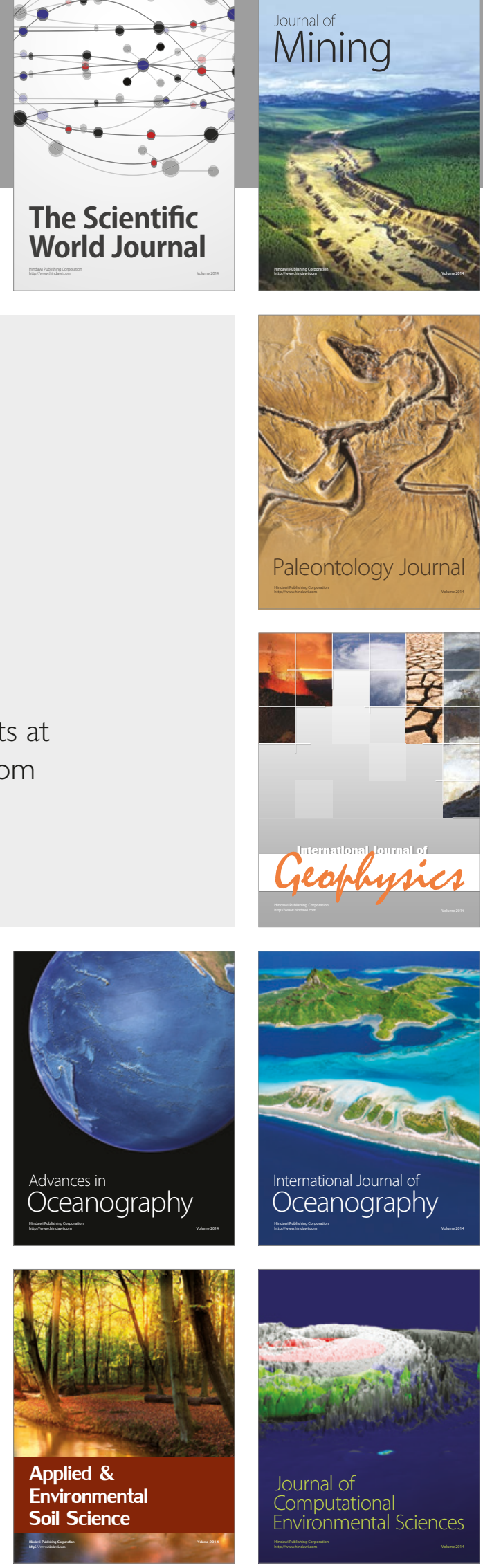